\title{
Development of free curved plate thickness measurement system
}

\section{- Improvement of interpolation for continuous path -}

\author{
Yurie OKUGAWA*, Naoki ASAKAWA* and Masato OKADA* \\ * Graduate School of Natural Science and Technology, Kanazawa University \\ Kakuma-machi, Kanazawa-shi, Ishikawa 920-1192, Japan \\ E-mail: yurie22@stu.kanazawa-u.ac.jp
}

Received 27 February 2014

\begin{abstract}
A method to generate a high-continuity thickness measuring path has been proposed in our own developed automatic system using laser displacement sensors and an industrial robot to measure and evaluate a free curved plate thickness. Besides the system generates the thickness measuring path on the basis of the point cloud data representing a workpiece shape, in this method, a quaternion is used to represent a change in the measuring posture with squad which is an interpolation algorithm. This paper presents a new method to determine the positions and the postures of the representative points for continuous interpolation. These positions and the postures are determined using 3D Configuration space(C-Space) consisting three parameters for representing the measuring posture. For details, the measurable postures are mapped in the C-Space on each measuring point. Then, the positions of the representative points are determined using a local maximum value of the distance between centroid coordinate of the measurable postures in adjacent points. Moreover, the postures of them are determined using the common postures of adjacent points or the distance from the origin of the C-Space. The continuity of the measuring path is evaluated from the change in the joint angle of the robot. Specifically, angular acceleration of the joint angle is used to evaluate for stabilization of the high-speed robot motion. The experimental result shows that the angular acceleration of the robot joint in generated path is smaller than that of the path generated by a conventional method.
\end{abstract}

Key words : Thickness measurement, Industrial robot, Path planning, Quaternion, Squad

\section{Introduction}

In general, thickness of sheet metal processed product with free curved surface is measured with point micrometer on the basis of the contact principle in the plastic processing field. However this measurement method is inappropriate to measure many points because it takes significant amount of time to measure (JSTP, 2000) and it is difficult to measure the whole of a workpiece with high density. Usually the several points that the operator has determined are most often measured to evaluate the workpiece. There is no appropriate system for measuring the thickness of a free curved plate with high density. Therefore, we proposed an automatic system for measuring and evaluating the thickness of free curved plates using laser displacement sensors and an industrial robot (Okugawa, et al., 2013a).

Although this system has been capable of measuring a workpiece with a relatively simple shape, it had collisions between the laser path and a workpiece with complicated shape, as a result of the geometrical limit for the measuring postures of the sensors. Then, it is necessary for the system to detect collisions and avoid these by changing the measuring posture through the use of CAD (Computer-Aided Design) data. However, the laser displacement sensors used in the system have measurement error characteristics that depend on the measuring posture. The system generates a measuring path considering the continuity of the measuring postures for stably speed up the measurement in addition to the above problems. We proposed a method to generate a measuring path with high continuity of the measuring postures by using a quaternion to represent a change in posture with an interpolation algorithm, called squad (spherical 
and quadrangle) (Shoemake, 1985) (Deloura, 2000) in the report (Okugawa, et al., 2013b).

In this paper, a new method is proposed to determine the positions and the postures of the representative points for appropriate interpolation. In addition, the validity of the proposed method was confirmed through evaluations of the continuity of a path depending on changes in the joint angle of a robot.

2. Outline of the measurement system

2.1 Configuration of the system

Figure 1 shows the configuration of the system. A dual-head thickness measuring unit composed of two laser displacement sensors, which are placed to share the same optical axis, measures the thickness of a workpiece. This unit uses two triangulation-based laser sensors (LK-G150, Keyence Co., reference distance of $150 \mathrm{~mm}$, spot diameter of $\phi 120 \mu \mathrm{m}$, and positioning accuracy of $\pm 0.04 \mathrm{~mm}$ ) as laser displacement sensors. The system uses a vertical articulated industrial robot (HP-6, Yasukawa Electric Co., payload of $6 \mathrm{~kg}$ and positioning accuracy of $\pm 0.08 \mathrm{~mm}$ ), as a workpiece positioning device.

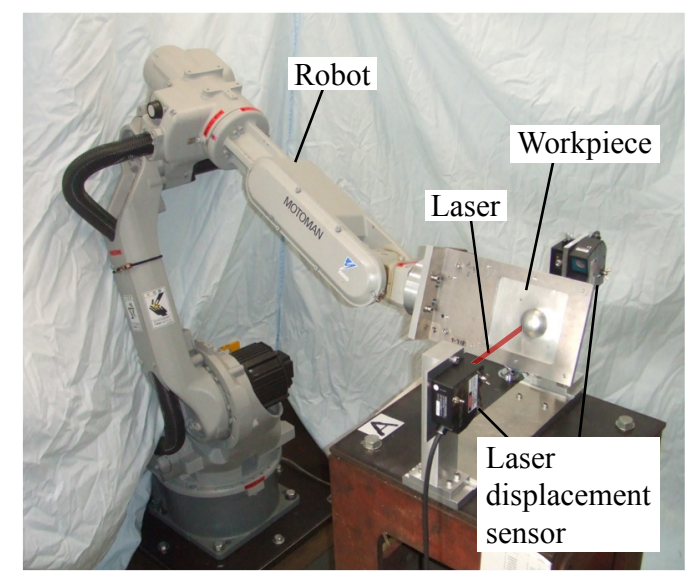

Fig. 1 Configuration of the developed system.

\subsection{Principle of measurement procedure}

The basic measurement procedure is as follows (Okugawa, et al., 2013a).

1) Measure the workpiece shape with a sensor. The result of the measurement is represented by a point cloud.

2) Generate a normal vector at each measuring point according to the workpiece shape.

3) Generate a series of postures for the robot to match the normal vector and the optical axis of the laser as a measuring path. A posture when the laser is vertically incident on the measuring surface is defined as a basic posture. A triangular area surrounded by the laser irradiation light, reflected light, and sensor is defined as the laser-path area.

4) Measure the workpiece thickness according to the measuring path.

\section{Evaluation of posture using C-Space}

In the case of a workpiece with complicated shape, it is possible to have collisions between the laser-path area and the workpiece because of the geometrical limit for the measuring postures of the sensors, as shown in Fig. 2. The system avoids collisions by changing the posture from the basic posture while remaining the same measuring position. Because we define a coordinate system $\left\{\mathrm{B}_{i}\right\}$ ( $i$-th measuring point) where the $x$-axis represents the normal direction of each measuring point and the $z$-axis represents the measuring process direction, as shown in Fig. 3, the measuring posture is represented by rotational angles $\gamma_{\mathrm{B} i}, \beta_{\mathrm{B} i}$, and $\alpha_{\mathrm{B} i}$ (roll, pitch, and yaw) around the $x_{\mathrm{B} i}, y_{\mathrm{B} i}$, and $z_{\mathrm{B} i}$ axes, respectively. The posture having $\gamma_{\mathrm{B} i}=\beta_{\mathrm{B} i}=\alpha_{\mathrm{B} i}=0^{\circ}$ represents basic posture. According to the previous research (Okugawa, et al., 2013a), in pitch, the measurement errors increase enormously in the case of $\beta_{\mathrm{B} i}$ is in the range of $-11^{\circ}$ to $-6^{\circ}$ as shown in Fig. 4 because of the increase in the regular-reflection component from the measuring surface. The 
postures in this range are defined as regular-reflection postures, and the system has no ability to measure where the posture has a pitch value in the regular-reflection postures. The postures in other areas of the above pitch, roll, and yaw angle range have an accuracy of $\pm 0.04 \mathrm{~mm}$ in the range of $-40^{\circ}$ to $40^{\circ}$.

We apply a 3D configuration space (C-Space) (Lozano-Perez, 1983) to deal with the geometric collisions and the measurement error characteristics in the same manner. The system uses a C-Space defined by rotational angles $\gamma_{\mathrm{A}}, \beta_{\mathrm{A}}$, and $\alpha_{\mathrm{A}}$ around the $x_{\mathrm{A}}, y_{\mathrm{A}}$, and $z_{\mathrm{A}}$ axes of coordinate system $\{\mathrm{A}\}$, where the $x$-axis represents the laser irradiation direction, and the $z$-axis represents the vertical direction defined as shown in Fig. 2, for each measuring point, to consider the continuity of the measuring postures. The postures that are collision free, regular-reflection free, and within the range of $\pm 40^{\circ}$ to the basic posture in the coordinate system $\left\{\mathrm{B}_{i}\right\}$ are mapped in the C-Space. The postures mapped in the C-Space and which are measured without collision with the nominal specification accuracy are called measurable postures. Figure 5 shows an example of measurable postures mapped in the C-Space.

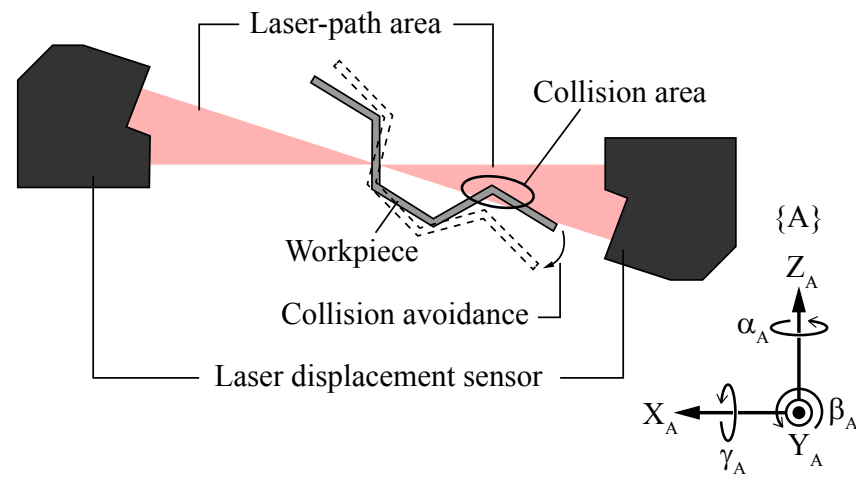

Fig. 2 Collision between laser-path area and workpiece.

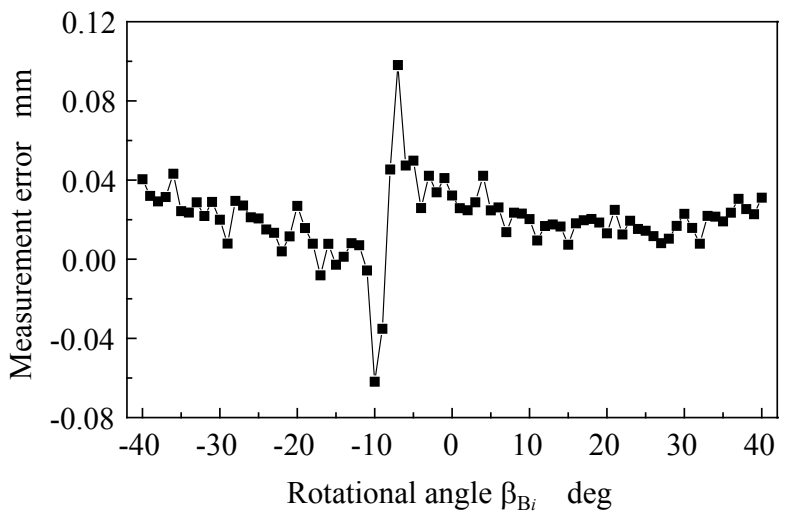

Fig. 4 Relationship between pitch angle and error.

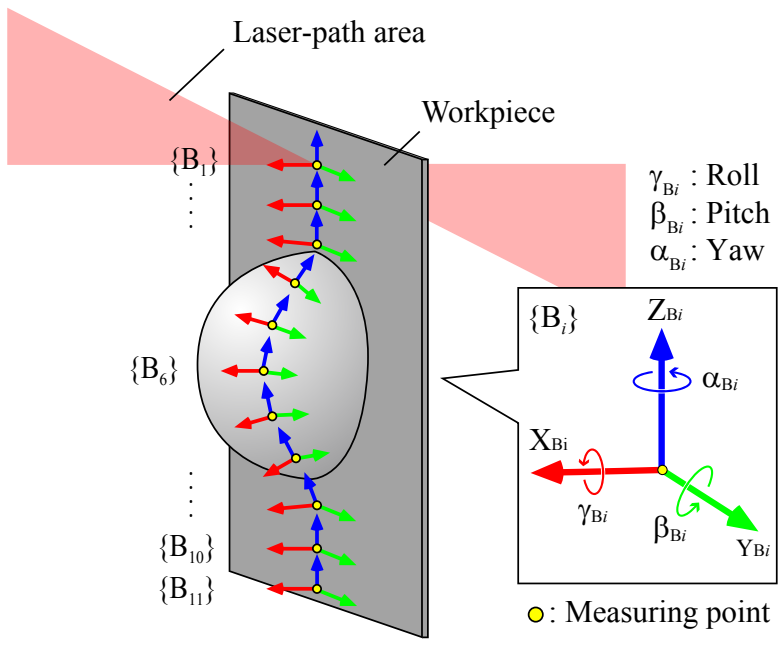

Fig. 3 Definition of coordinate system $\left\{\mathrm{B}_{i}\right\}$.

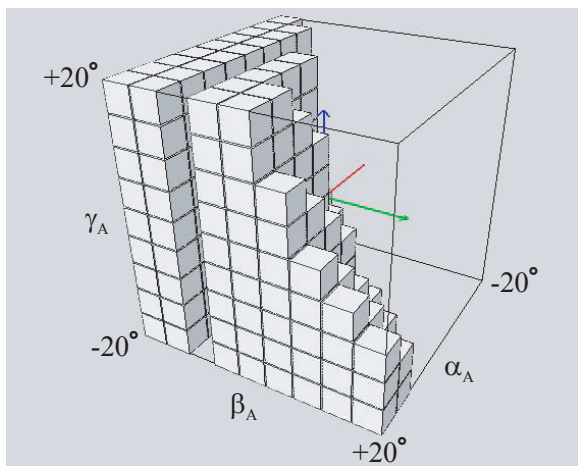

Fig. 5 An examples of measurable postures mapped in the C-Space.

\section{Smooth interpolation of measuring postures by use of squad}

The system determines the measuring posture by selecting an arbitrary posture from the measurable postures mapped in the C-Space on each measuring point. Although the representation of a posture by the use of the C-Space is appropriate for representing a static condition, it is difficult to select the most continuous set of postures in multiple C-Spaces. In this study, to generate a measuring path with a high continuity of measuring postures, we apply a quaternion used in 3D computer animations (Lee, et al., 2007) and posture calculations for a flying object (Markley, 2000) to represent a change in posture, by using the popular interpolation algorithm called squad (Shoemake, 1985) (Deloura, 2000). Squad is the only algorithm that interpolates serial postures more than three points to define a path continuously. Quaternion $q$ has four elements, a set of a scalar element $w$ and a $3 \mathrm{D}$ vector element $\mathbf{v}=(x, y, z)$. In addition, unit quaternions are used for representing a change in posture. 


$$
q=[w, x, y, z]=[w, \mathbf{v}] \quad(|q|=1)
$$

Given the quaternion as a 4D vector, a formula for squad from $q_{n}$ to $q_{n+1}$, with parameter $t$ moving from 0 to 1 , can be obtained as

$$
\operatorname{squad}\left(t ; q_{n}, q_{n+1}, a_{n}, a_{n+1}\right)=\operatorname{slerp}\left(2 t(1-t) ; \operatorname{slerp}\left(t ; q_{n}, q_{n+1}\right), \operatorname{slerp}\left(t ; a_{n}, a_{n+1}\right)\right)
$$

Slerp (spherical liner interpolation) is an algorithm that interpolates between the continuities of two postures.

$$
\operatorname{slerp}\left(t ; q_{n}, q_{n+1}\right)=\frac{q_{n} \sin (\theta(1-t))+q_{n+1} \sin (t \theta)}{\sin \theta}
$$

Constant factor of the quaternion is equal to the product of a constant and a 4D vector. Here, $\theta$ is the angle between $q_{n}$ and $q_{n+1}$, as shown in Fig. 6 and defined as

$$
\theta=\cos ^{-1}\left(q_{n} \bullet q_{n+1}\right)
$$

Slerp resolves liner interpolation when $\sin \theta$ approaches 0 as $\left|q^{\circ} \cdot q_{1}\right|$ approaches 1 . The scalar product of quaternion $q_{0}$ and $q_{1}$ is calculated as

$$
q_{0} \bullet q_{1}=w_{0} w_{1}+x_{0} x_{1}+y_{0} y_{1}+z_{0} z_{1}
$$

In addition, $a_{n}$ is defined as follows:

$$
a_{n}=q_{n} \exp \left[-\frac{\log \left(q_{n}^{-1} q_{n-1}\right)+\log \left(q_{n}^{-1} q_{n+1}\right)}{4}\right]
$$

$a_{n}$ is the quaternion sequence that the derivative of squad is continuous on control point, with parameter $t=0$ and 1 . In other words, if $S_{n}(t)=\operatorname{squad}\left(t, q_{n}, q_{n+1}, a_{n}, a_{n+1}\right), a_{n}$ is the quaternion sequence that $S_{n}^{\prime}(0)=S_{n-1}^{\prime}(1)$ in all $n$. for calculation $a_{n}$, some operations of the quaternion are required. The reciprocal of $q$ is defined as

$$
q^{-1}=\frac{[w,-\mathbf{v}]}{|q|^{2}}
$$

The multiplication of quaternion $q_{0}$ and $q_{1}$ is defined as

$$
\begin{aligned}
q_{0} q_{1} & =\left[w_{0}, \mathbf{v}_{0}\right]\left[w_{1}, \mathbf{v}_{1}\right] \\
& =\left[w_{0} w_{1}-\mathbf{v}_{0} \bullet \mathbf{v}_{1}, w_{0} \mathbf{v}_{1}+w_{1} \mathbf{v}_{0}+\mathbf{v}_{0} \times \mathbf{v}_{1}\right] \\
& \left.=\left[\begin{array}{c}
w_{0} w_{1}-x_{0} x_{1}-y_{0} y_{1}-z_{0} z_{1} \\
w_{0} x_{1}+x_{0} w_{1}+y_{0} z_{1}-z_{0} y_{1} \\
w_{0} y_{1}+y_{0} w_{1}+z_{0} x_{1}-x_{0} z_{1} \\
w_{0} z_{1}+z_{0} w_{1}+x_{0} y_{1}-y_{0} x_{1}
\end{array}\right]\right]
\end{aligned}
$$

For calculation of a logarithm, an variable $\sigma$ which is equal to half of the rotational angle $\theta / 2$ is defined and a quaternion $p$ is reformulated using $\sigma$ and a $3 \mathrm{D}$ vector $\mathbf{u}=\left(x_{u}, y_{u}, z_{u}\right)$ with unit length as follows:

$$
\begin{aligned}
\sigma & =\theta / 2 \\
\|\mathbf{u}\| & =1 \\
p & =[\cos \sigma, \mathbf{u} \sin \sigma] \\
& =\left[\cos \sigma, x_{u} \sin \sigma, y_{u} \sin \sigma, z_{u} \sin \sigma\right]
\end{aligned}
$$

Here, the logarithm of $p$ is defined as follows:

$$
\begin{aligned}
\log p & =\log (\cos \sigma, \mathbf{u} \sin \sigma) \\
& \equiv[0, \sigma \mathbf{u}]
\end{aligned}
$$

$\log p$ is not basically the quaternion with unit length. If quaternion $r$ is defined as $[0, \sigma \mathbf{u}]$, the exponent of $r$ is defined as 
follows:

$$
\begin{aligned}
r= & {[0, \sigma \mathbf{u}] } \\
= & {\left[0, \sigma x_{u}, \sigma y_{u}, \sigma z_{u}\right] } \\
\exp r & =\exp ([0, \sigma \mathbf{u}]) \\
& =[\cos \sigma, \mathbf{u} \sin \sigma]
\end{aligned}
$$

$\exp r$ is the quaternion with unit length. From the above, squad needs $q_{n-1}, q_{n}, q_{n+1}$, and $q_{n+2}$ for the interpolation between $q_{n}$ and $q_{n+1}$. If the required quaternions for squad include that of the end point of the path, copied quaternions are given. We assign 0 to $w$ and a $3 \mathrm{D}$ vector with unit length representing the measuring posture to $\mathbf{v}$ in the quaternion shown in Eq. (1). In this paper, existing measuring points are used for interpolation. In other words, the postures interpolated are on the existing points. Figure 7 shows an example of the 3D vector interpolation, with arrow vectors showing representative directions. In this figure, 4 points out of 20 points are determined as the representative points and the initial postures are given. Because the measuring direction of the system is unidirectional, as shown in Fig. 8, a set of measuring points in a column is defined as a measuring-path element. In this paper, the system considers the continuity in a measuring-path element.

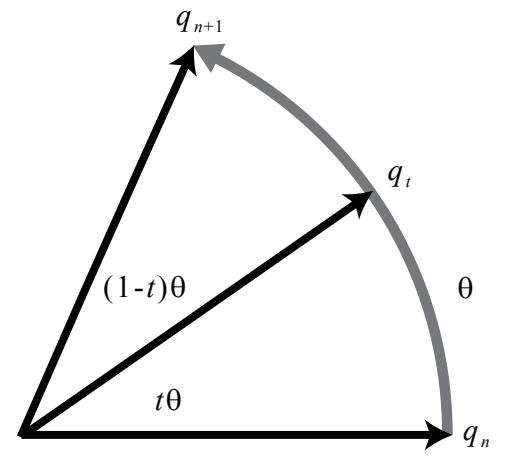

Fig. 6 Interpolation of rotation with slerp.

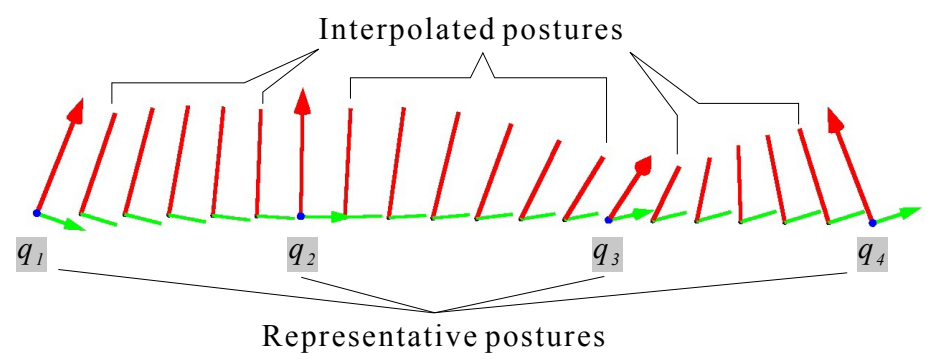

Fig. 7 Interpolation of posture with squad.

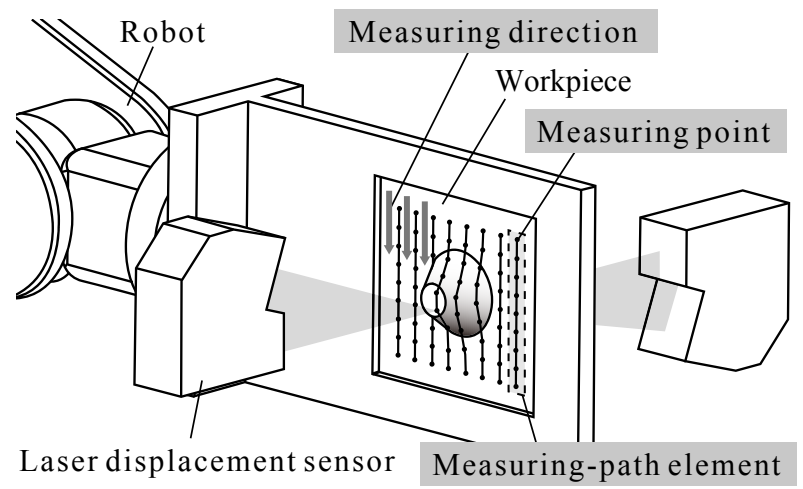

Fig. 8 Measuring direction in this study.

\section{Determination of representative points and initial postures}

In this study, the system determines the positions and postures of the representative points so that it reduces the number of the representative points wherever possible for generation of a high-continuity measuring path using the interpolation algorithm. Candidates for representative point are measuring point and that for initial posture area measurable postures mapped in the C-Space. Thus, the postures on the measuring points except the representative points are determined by use of squad with interpolation parameter $t$ depending on the number of the measuring points between the representative points. Firstly, the system defines the representative points from the starting point and the ending point of a measuring-path element. Secondly, it calculates the centroid coordinate of the measurable postures 
mapped in the C-Space on each point in the measuring-path element and the distance between the centroid coordinates of adjacent points, respectively. In consequence, it obtains the points having the local maximum value of the distance. Then it compares the measurable postures mapped in the C-Space on the points around the points having the local maximum value, and determines the other representative points in the following two methods:

1) The point having the local maximum value is defined as a representative point when the three measurable postures have the common postures.

2) The points around the points having the local maximum value are defined as representative points when the three measurable postures have no common postures.

The measuring posture on a representative point is defined as an initial posture. It is defined as the initial posture that the posture with the centroid of the common postures in 1), the posture with the minimum distance from the origin of the C-Space in 2), respectively.

\section{Generation of measuring path}

The procedure to generate a measuring path using squad is as follows:

1) The system detects collisions at the basic posture on each measuring point.

2) It maps measurable postures in the C-Space on each measuring point in the measuring-path element, including the points where collisions have been detected in 1), as shown in Fig. 5.

3) It determines the representative points and the initial postures.

4) It interpolates between the initial postures on the representative points determined in 3) by using squad. It verifies whether the interpolated postures are measurable postures. If there are unmeasurable postures, it defines the points as new representative points and interpolates again. It repeats this process until all the postures fit into the measurable postures. It generates the measuring path in the basic postures if there are no collisions in a measuring-path element.

\section{Experiment}

A measuring experiment was carried out to verify the validity of the generated measuring path. Table 1 lists the experimental conditions, and Fig. 9 shows the workpiece and the measured area. The C-Space was generated within a range of $\pm 20^{\circ}$ at intervals of $5^{\circ}$, considering the calculation cost to detect collisions. Figure 10 shows the thickness distribution map. It shows that the system could measure the thickness of the workpiece at all the measuring points. In general, the thickness of the machined part is expected to have been small, and the thickness measurement by the system reflects this tendency. Figure 11 shows the measurement errors of the system in reference to the measurement with a point micrometer in a measuring-path element. All the errors were within the range of the designed accuracy of the system. Figure 12 shows one column of the measuring path on the red line in Fig. 9. Path1 shows the generated path by the proposed method, and path2 shows that by the simpler method for comparison. Path2 is generated by use of the same interpolation algorithm squad with the same representative positions but has the different initial postures as path1. The initial postures of it have the minimum distance from the origin of each C-Space.

Next, we evaluate the continuity of the measuring path from change in the joint angles of the robot. To stabilize a high-speed robot motion, it is necessary to make angular accelerations of the joint angles smaller without sudden change in posture. Figure 13 shows the values corresponding to the changes in angular displacement, angular velocity, and angular acceleration on sixth axis of the robot having the largest motions in the joints in a measuring-path element shown in Fig. 12. Figure 13(a) shows that although the variation range of path2 is smaller, it is discontinuous in the middle of the path. The discontinuous motion of path2 is due to additional representative points that are generated after unmeasurable postures have been found at the first interpolation. On the other hand, interpolation is performed only once in path 1 and unmeasurable postures are not generated at the interpolation in path1. Figure 13(c) shows that the maximum value of path1 is lower besides path 2 makes large changes in the value. These data lead us to the conclusion that the system could measure the thickness of the workpiece with complicated shape, and the measuring path generated by the proposed method had high continuity for the measuring postures. 
Table 1 Experimental conditions.

\begin{tabular}{c|c}
\hline \hline Material & Alminum (A1050) \\
Size & $125 \times 135 \mathrm{~mm}$ \\
Thickness & $0.5 \mathrm{~mm}$ \\
Measurement range & $46 \times 46 \mathrm{~mm}$ \\
Measurement interval & $2 \mathrm{~mm}$ \\
Number of measurement points & 529 \\
Shape & Twin frustums of a cone \\
\hline
\end{tabular}

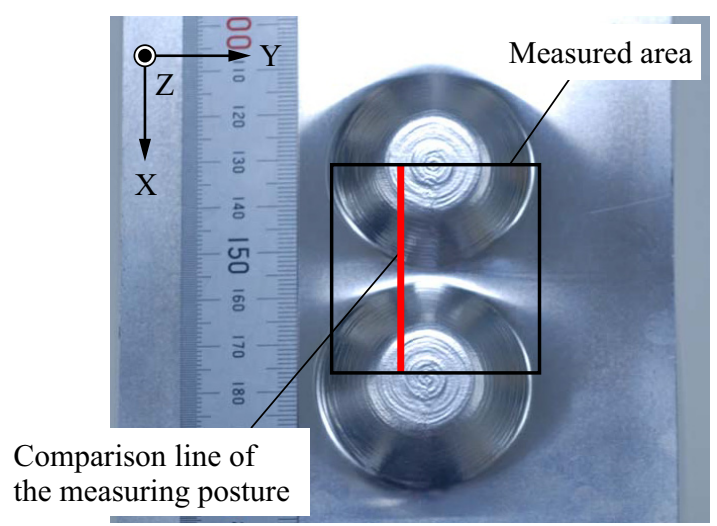

Fig. 9 Workpiece to be measured.

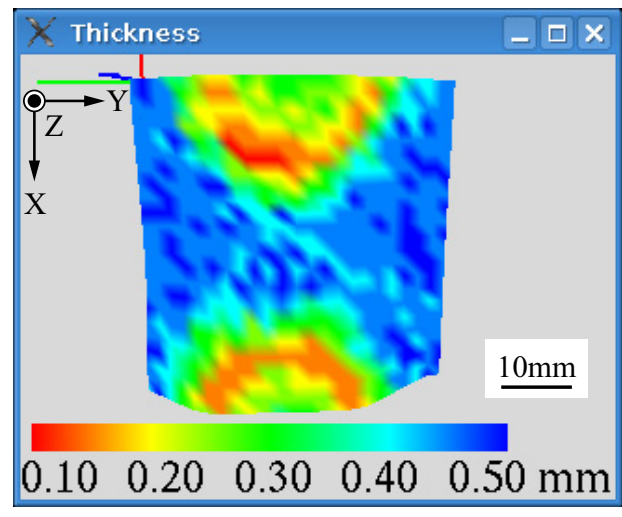

Fig. 10 Thickness distribution map.

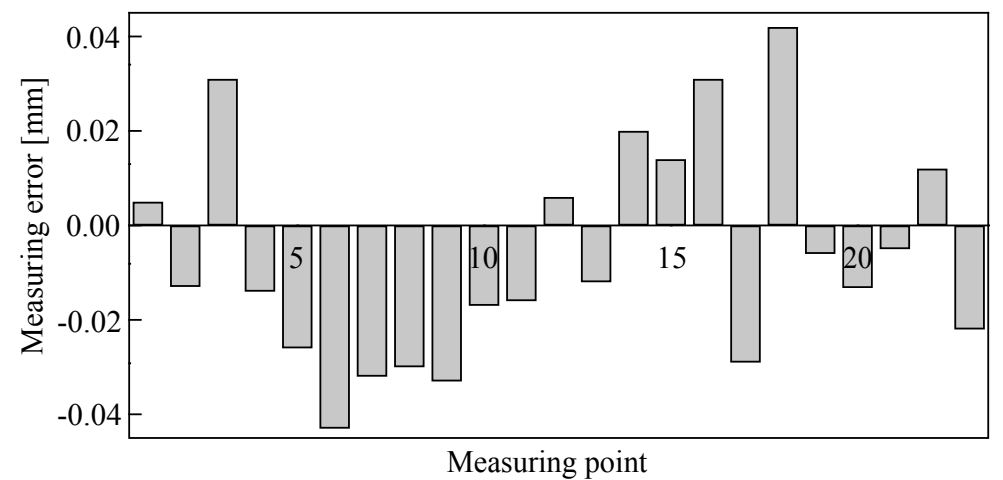

Fig. 11 Measurement errors in reference to measurements with a point micrometer in a measuring-path element.

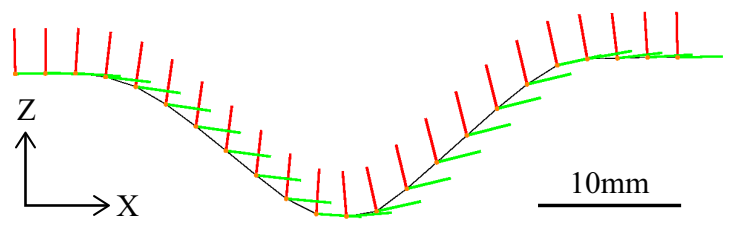

(a) Path1.

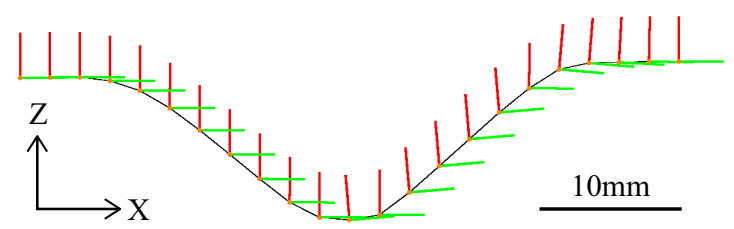

(b) Path2.

Fig. 12 Measuring path. Orange points show measuring position. Led lines show laser irradiation direction. Green lines show measuring direction. Black line show workpiece shape (Red line in Fig. 9).

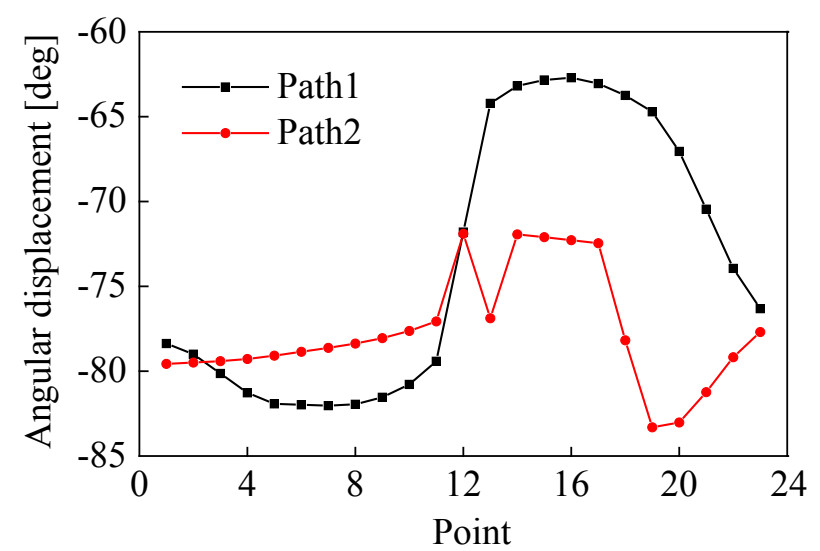

(a) Angular displacement.

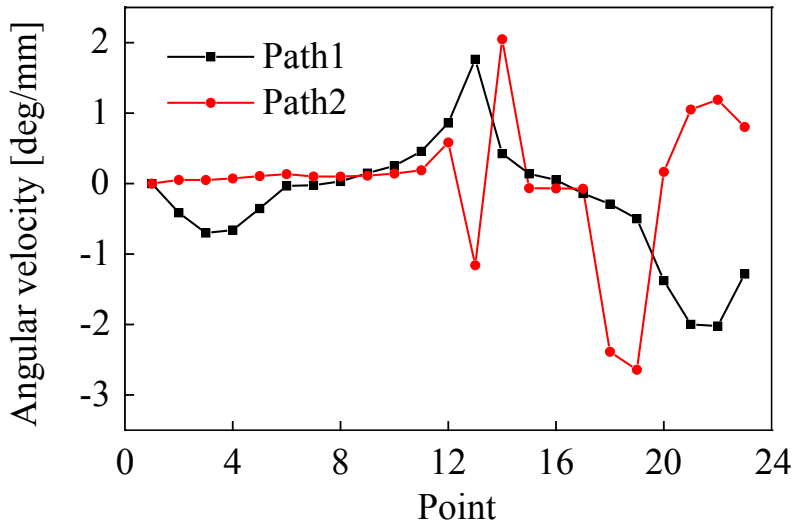

(b) Angular velocity. 


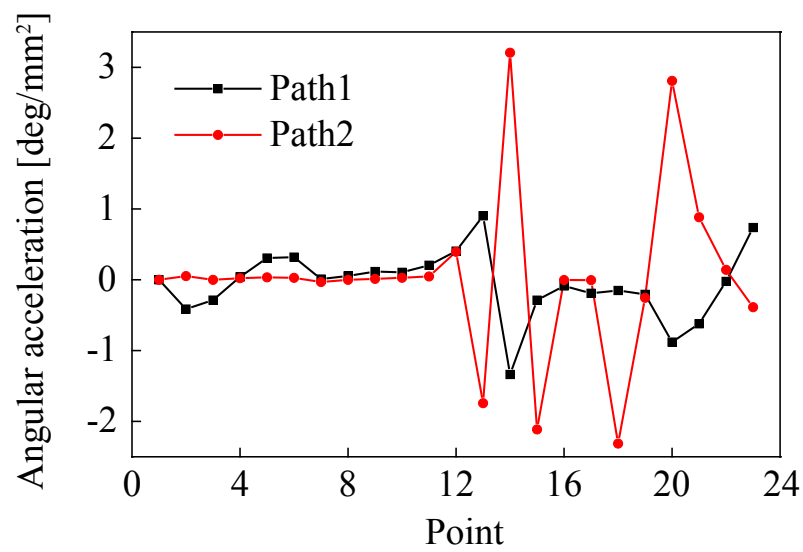

(c) Angular acceleration.

Fig. 13 Comparison of change in the sixth axis of the robot with conventional method in a measuring-path element.

\section{Conclusion}

This study proposed a method for determining the representative points and the initial postures to generate a thickness measuring path with high continuity of the measuring postures using squad interpolation algorithm. From the experimental results, this paper can be summarized as follows:

1) From the distance between the centroid coordinate of the measurable postures mapped in the C-Space of adjacent measuring points and the common postures of them, the system could determine the positions and postures of the representative points for appropriate interpolation using squad.

2) In the experiment to measure the thickness of a workpiece with complicated shape using the generated measuring path, the system could measure the thickness within the designed accuracy range in all measuring points.

3) The evaluation results with changes in the joint angles of a robot showed that the generated path had high continuity.

\section{References}

Deloura, M., Game Programming Gems, trans. Kawanishi, H., Karino, T., Born Digital, Inc. (2000), (in Japanese).

Lee, S. -H., Kwon, K. -R., 3D Keyframe Animation Watermarking based on Orientation Interpolator, IEICE Transactions on Information and Systems, Vol.E90-D, Issue 11 (2007), pp. 1751-1761.

Lozano-Perez, T., Spatial Planning: A Configuration Space Approach, IEEE Transactions on Computers, Vol.C-32, Issue 2 (1983), pp.108-120.

Markley, F. L., Mortari, D., Quaternion Attitude Estimation using Vector Observations, Journal of the Astronautical Sciences, Vol.48, Issue 2-3 (2000), pp.359-380.

Okugawa, Y., Asakawa N., Okada M., Development of a System for Measuring the Thickness of Free Curved Plates -Measurement Posture Planning Using C-Space-, International Journal of Automation Technology, Vol.7, No.5 (2013a), pp.593-600.

Okugawa, Y., Asakawa, N., Okada, M., Development of Orthros, Free Curved Plate Thickness Measurement System -Generation of Path Considering of Postures-, Proceeding of the 17th International Conference on Mechatronics Technology (2013b), pp.132-136.

Shoemake, K., Animating Rotation with Quaternion Curves, Computer Graphics (ACM), Vol.19, Issue 3 (1985), pp. 245-254.

The Japan Society for Technology of Plasticity, Plain Press Forming, The Nikkan kogyo shinbun (2000), (in Japanese). 